\title{
ANÁLISE DE RISCOS E CONSEQUÊNCIAS EM UMA USINA SUCROALCOOLEIRA
}

\author{
M. P. A. MARIN ${ }^{1, *}$, G.M. DE MELO ${ }^{1}$, G.B.H. DE PAULO ${ }^{1}$, L.F.M. LABATE ${ }^{1}$, \\ T.B. MIRANDA ${ }^{1}$, V. SCHINCARIOL ${ }^{1}$ \\ *E-mail: marimari@fei.edu.br
}

\begin{abstract}
RESUMO: A indústria sucroalcooleira possui grande influência no Brasil. Porém, assim como todos os processos, há perigos e podem ocorrer acidentes. Mesmo com o avanço tecnológico, existe o perigo em cada etapa do processo. Este trabalho tem como objetivo identificar e analisar os riscos existentes da indústria sucroalcooleira, dividindo o processo em blocos e identificando os principais, além de levantar um inventário de substâncias perigosas usadas no processo, mais principalmente o álcool anidro e o hidratado e o açúcar, devido ao grande volume no processo e suas características físico-químicas. Com esses dados, um histórico de acidentes foi levantado, mostrando que $90 \%$ dos acidentes envolvendo o etanol, ocorre no transporte rodoviário $e, 2 \%$ na indústria $e$ no armazenamento. Dessa forma, a análise de riscos foi realizada nos armazenamentos dos produtos devido às suas periculosidades e quantidades, no transporte devido ao histórico $e$ na destilação devido a sua alta importância no processo. Foram aplicados as APPs, AAE e Hazops como métodos qualitativos de análise. O software Phast ${ }^{\circledR}$ mostrou, como análise quantitativa, que o maior raio de alcance foi de 169,9 metros para o evento de liberação de Etanol pela ruptura do reservatório de $10.000 \mathrm{~m}^{3}$. Também com o auxílio do software Phast Risk $^{\circledR}$, foram obtidos os riscos social e individual. Os resultados apontam que as fatalidades ocorrerão dentro da indústria apenas, indicando somente risco individual. Esse risco atinge o máximo de 1E-04, considerado intolerável pela CETESB.
\end{abstract}

PALAVRAS-CHAVE: Análise de risco; Identificação de perigos; Hazop; APP; AAE

\section{INTRODUÇÃO}

O desenvolvimento da sociedade deve-se aos avanços tecnológicos, os quais originam produtos que permitem o estilo de vida de hoje e que a cada dia é revolucionado. A grande maioria destes produtos, como medicamentos, detergentes, tintas, plásticos, combustíveis, dentre outros, vêm de diferentes processos industriais de natureza química. O homem é o grande responsável por essa alteração industrial, o qual possui a inteligência e o raciocínio para as mudanças do dia a dia. Mas neste papel inclui-se a responsabilidade de gerenciar os efeitos, causas e consequências desta alteração, ou seja, os riscos que envolvem todo este processo (REVISTA BRASILEIRA DE ENGENHARIA QUÍMICA, 2008).

Com a Revolução Industrial, gerou-se um grande aumento na produção industrial e também teve o início e aumento de acidentes industriais. A gravidade dos acidentes pode 
variar entre pequenos vazamentos até grandes explosões, mas a análise de perigos e riscos somente se tornou relevante quando o dramático acidente de Bhopal na Índia ocorreu em 1980 (SOUZA, 200-).

Em um âmbito geral, o conceito de segurança pode ser entendido como um conjunto de ações que visam a proteção contra um determinado risco. Já a segurança de processo lida com um conjunto de ações que objetivam minimizar qualquer evento com potencial de provocar danos a equipamentos e estruturas ou até mesmo danos e lesões às pessoas que trabalham no local do evento ou vivem perto do mesmo (COSTA, 2015).

Segundo Souza (1995), analisar um risco é identificar, discutir e avaliar as possibilidades de ocorrência de acidentes, na tentativa de se evitar que estes eventos aconteçam e, caso ocorra, identificar as alternativas que tornam mínimos os danos subsequentes a estes acontecimentos.

No Brasil, segundo Lima et al. (2001), a indústria sucroalcooleira possui alta importância econômica devido à produção de açúcar, sendo o país o maior exportador do mundo, e etanol, competidor dos combustíveis fósseis. Atualmente o Brasil é o segundo maior produtor de álcool do mundo. Desde 1978, a CETESB (2015), relata acidentes na indústria sucroalcooleiras. Acidentes estes envolvendo substâncias como álcool anidro e hidratado, melaço, $\mathrm{SO}_{2}$, leite de cal, entre outros.

Tendo em vista a necessidade de entender melhor estes acidentes, este trabalho tem como objetivo estudar e analisar os perigos e riscos e suas consequências numa indústria sucroalcooleira que use como matéria-prima a cana-de-açúcar. Para tal estudo serão aplicadas técnicas específicas como identificação e separação do processo em blocos principais, identificação dos perigos em cada bloco e por fim modelos específicos para se avaliar quantitativamente as consequências em um ou mais blocos.

\section{MATERIAIS E MÉTODOS}

\subsection{Método de Análise}

Tendo como base o processo de uma indústria sucroalcooleira, foram seguidos os seguintes passos, a partir o manual de técnicas de avaliação de perigos industriais do banco mundial (THE WORLD BANK, 1990): o fluxograma de processo foi dividido em blocos em função das operações ou atividades realizadas (como filtração, destilação, secagem, armazenamento).

Um histórico de acidentes foi realizado para assim ter-se conhecimento de quais tipos de perigos e onde se tem o maior número de acidentes, ou seja, em quais partes do processo os acidentes ocorreram; inventário de materiais perigosos na planta e suas localizações foram levantados; quais os blocos apresentam maior vulnerabilidade; foram avaliados os dados de toxicidade, inflamabilidade e das propriedades físico-químicas das substâncias, selecionando quais serão avaliadas qualitativamente, aplicando métodos para identificação de perigos e desvios de condições normais de operação, utilizando-se de técnicas específicas como APP, HAZOP ou AAE. 
Utilizou-se o software Phast para avaliação das consequências de alguns cenários levantados e foram interpretados os resultados e indicadas medidas corretivas ou mitigadoras.

\subsection{Técnicas Qualitativas de Análise}

Análise preliminar de perigos (APP): A aplicação desse método é feita através do preenchimento de uma planilha padrão para cada subsistema da instalação, na qual são identificados os perigos, causa, os efeitos (consequências) e as categorias de severidades correspondentes das consequências, bem como as observações e recomendações pertinentes aos perigos identificados, devendo os resultados serem apresentados em planilha padronizada.

Hazop: A aplicação de Hazop (Hazard and operability) é baseada em perguntas estruturadas e sistemáticas, usando um conjunto de "palavras guia" aplicadas a alguns pontos críticos que podem gerar desvios no sistema em estudo (nós de estudos). Estas palavras criam desvios dos padrões operacionais que são analisados em relação às causas e consequências. A fim de reduzir ou eliminar as probabilidades de ocorrência desses desvios, recomendações são propostas de acordo com a necessidade do sistema. Como a execução do Hazop é muito sistemática e estruturada, são necessários profissionais da área a ser aplicada assim como informações sobre o processo, da planta, os instrumentos, o empreendimento, todas detalhadas e atualizadas (RODRIGUES, 2009).

Análise de árvore de eventos (AAE): A aplicação dessa técnica parte de um evento e estuda as probabilidades resultantes do mesmo. Essas probabilidades são "caminhos" ou eventos secundários nos quais podem gerar mais probabilidades, ou seja, mais "caminhos". Como resultado, será gerado uma árvore na qual é possível calcular as probabilidades das consequências de cada evento. Normalmente avaliam-se duas alternativas para cada caso (sim/não) mas é possível haver mais de duas opções para cada evento (RAUSAND, 2005).

\subsection{Técnicas Quantitativas de Análise}

Iniciada a partir das simulações de vazamentos, foram levantadas hipóteses acidentais que estão associadas ao etanol anidro, um líquido inflamável, nas linhas de transferência, tanques de armazenamento e caminhões-tanque. Para estimar os efeitos físicos e avaliar as vulnerabilidades para as hipóteses que eventualmente venham a ocorrer durantes as operações da usina, é utilizado o Phast Risk ${ }^{\circledR}$ (Process Hazard Analysis Software Tools), versão 6.7 de 2012, desenvolvido pela DNV-Technica. O software não é capaz de analisar sólidos, portanto não é possível a análise quantitativa do açúcar.

O Phast Risk ${ }^{\circledR}$ é um software utilizado para avaliar potenciais situações de perigo à vida e ao meio ambiente, assim como quantificar suas severidades. Ele tem a capacidade de simular o progresso de um incidente potencial desde o seu vazamento inicial através da formação de uma nuvem ou poça, até sua dispersão, aplicando automaticamente os modelos de dispersão adequados ao fenômeno analisado.

\section{RESULTADOS E DISCUSSÕES}

Com base em um processo de uma sucroalcooleira, o fluxograma da Figura 1 foi 
desenvolvido.

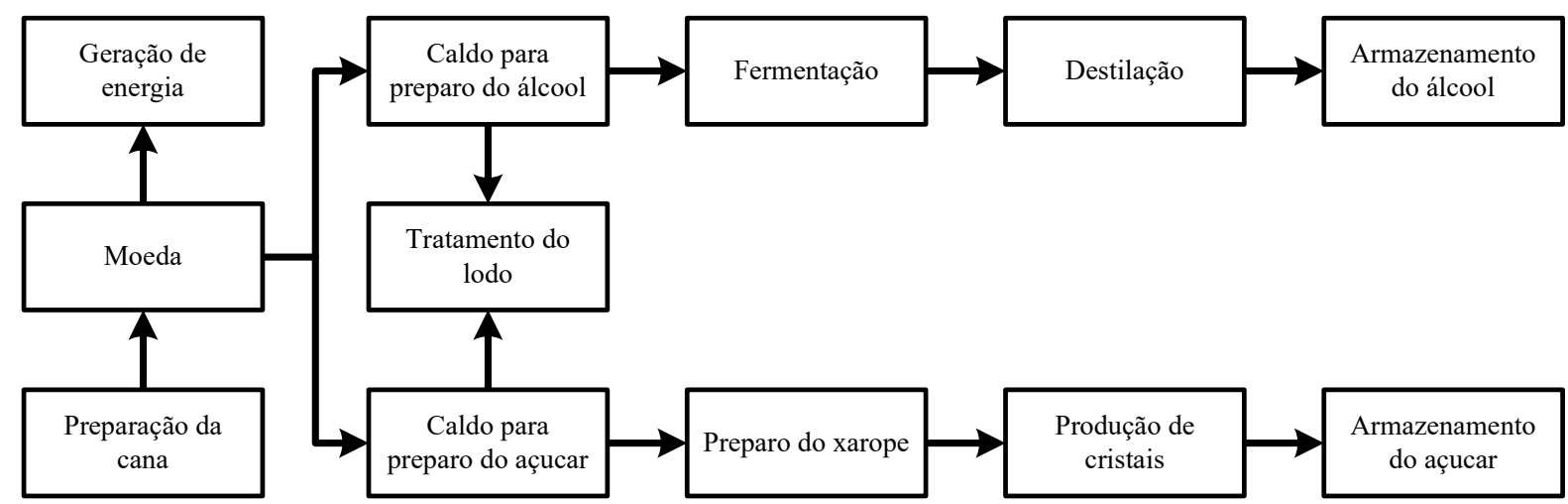

Figura 1 - Divisão em blocos do processo industrial.

Tendo como base o processo, o levantamento de substâncias perigosas determinou-se que o etanol é o foco das análises por ser mais perigoso e se apresentar em grandes quantidades em comparação com as outras substâncias. O levantamento também indicou a periculosidade do açúcar, justificando seu estudo. A pesquisa de históricos de acidentes revelou que grande parte dos acidentes envolvendo álcool está em acidentes rodoviários (90\%), envolvendo capotamentos (12\%), colisões (25\%) e tombamentos (35\%) e apenas $2 \%$ na indústria. A Tabela 1 mostra as porcentagens de acidentes para os álcoois anidro e hidratado na indústria e no transporte rodoviário.

Tabela 1 - Porcentagens dos acidentes ocorridos com álcoois hidratado e anidro na indústria e no transporte rodoviário

\begin{tabular}{ccccc}
\hline \multirow{2}{*}{ Acidente } & \multicolumn{2}{c}{ Álcool anidro } & \multicolumn{2}{c}{ Álcool hidratado } \\
\cline { 2 - 5 } & Indústria & Rodoviário & Causas & Rodoviário \\
\hline Colisão/Choque & $25 \%$ & $28 \%$ & $27 \%$ & $32 \%$ \\
Extravasamento & $4 \%$ & $2 \%$ & - & - \\
Tombamento & $35 \%$ & $38 \%$ & $25 \%$ & $30 \%$ \\
Capotamento & $12 \%$ & $13 \%$ & $9 \%$ & $11 \%$ \\
Descarrilamento & $4 \%$ & - & $2 \%$ & - \\
Falha operacional & $2 \%$ & $2 \%$ & $2 \%$ & - \\
Falha mecânica & $6 \%$ & $6 \%$ & $4 \%$ & $2 \%$ \\
Tubulação & $2 \%$ & - & - & - \\
Transbordo & - & - & $2 \%$ & - \\
Abalroamento & - & - & $2 \%$ & $2 \%$ \\
Incêndio & - & - & $4 \%$ & $2 \%$ \\
Tanque & - & - & $2 \%$ & $14 \%$ \\
Outra & $8 \%$ & $9 \%$ & $17 \%$ & $2 \%$ \\
Não identificada & $2 \%$ & $2 \%$ & $4 \%$ & \\
\hline
\end{tabular}

Dessa forma, foram feitas análises qualitativas para os armazenamentos de etanol anidro e hidratado por consequência suas grandes quantidades, para o transporte de etanol devido ao histórico e para o armazenamento de açúcar por causa das consequências. Também se realizou uma análise na torre de destilação por sua importante função no processo. 
Foram realizados APPs para os tanques de armazenamento de álcool e açúcar, HAZOP para a torre de destilação e uma AEE para vazamento de etanol, procurando identificar causas de eventos, seus efeitos e recomendações a fim de minimizar o perigo ou mitigar as consequências. Na Tabela 2, encontram-se os cenários analisados e quais perigos estão relacionados.

Tabela 2 - Cenários usados nas análises de risco qualitativas e seus perigos

\begin{tabular}{|c|c|}
\hline Cenário & Perigo \\
\hline Grande liberação de etanol na linha de 3" desde a produção até os tanques passando pelas bombas & VG \\
\hline Pequena liberação de etanol na linha de 3" desde a produção até os tanques passando pelas bombas & VM \\
\hline Liberação de Etanol pela ruptura do reservatório de $10,000 \mathrm{~m}^{3}$ & VG \\
\hline Liberação de Etanol pela ruptura do reservatório de $5.000 \mathrm{~m}^{3}$ & VG \\
\hline Liberação de Etanol pela ruptura dos reservatórios de $3.000 \mathrm{~m}^{3}$ & VG \\
\hline $\begin{array}{l}\text { Grande liberação de Etanol líquido na linha de 3" desde os tanques até as CTs passando pelas } \\
\text { bombas }\end{array}$ & VG \\
\hline $\begin{array}{l}\text { Pequena liberação de Etanol líquido na linha de 3" desde os tanques até as CTs passando pelas } \\
\text { bombas }\end{array}$ & VM \\
\hline Grande liberação de Etanol líquido no mangote de 3" & VG \\
\hline Pequena liberação de Etanol líquido no mangote de 3" & VM \\
\hline $\begin{array}{l}\text { Liberação de Etanol devido a ruptura de caminhão-tanque/carreta (Carreta de capacidade para } 28 \mathrm{~m}^{3} \\
\text { de etanol) }\end{array}$ & VG \\
\hline Curto circuito ou faíscas eletrostáticas no armazém de açúcar & IN \\
\hline Fonte de ignição para pó em suspensão em ambiente confinado & EX \\
\hline Colisão ou choque do caminhão durante o transporte de Etanol & EX \\
\hline Aumento da pressão do tanque Incêndio externo por causas naturais (raios) & EX \\
\hline $\begin{array}{l}\text { Falha na bomba, válvula de alimentação, válvula de saída ou no controlador de nível na torre de } \\
\text { destilação }\end{array}$ & CL \\
\hline $\begin{array}{l}\text { Menor fluxo de alimentação, falha nas válvulas de saída ou falha no controlador de nível na torre de } \\
\text { destilação }\end{array}$ & PRV \\
\hline $\begin{array}{l}\text { Maior fluxo de alimentação, falha nas válvulas de saída ou falha no controlador de nível na torre de } \\
\text { destilação }\end{array}$ & $\mathrm{IC}$ \\
\hline Falha em processo anterior à destilação ou contaminação de uma linha do processo & PFE \\
\hline Falha na válvula de saída ou menor geração de vapor no refervedor & PFE \\
\hline Ruptura da tubulação do refervedor, maior geração de vapor no refervedor ou falha no condensador & PFE \\
\hline Falha no refervedor ou falha no isolamento da coluna & PFE \\
\hline Falha no refervedor ou diminuição da vazão de alimentação & PFE \\
\hline
\end{tabular}

Os cenários da APP foram aplicados no software Phast Risk ${ }^{\circledR}$ para a determinação dos raios de perigo que cada hipótese resulta. A Tabela 3 mostra as hipóteses aplicadas, as maiores distâncias e qual condição estão relacionadas.

A análise mostrou que o maior raio é 169,9 metros para a liberação de Etanol pela ruptura do reservatório de $10.000 \mathrm{~m}^{3}$. Ou seja, dentro desse raio, o efeito de explosão com ignição tardia gera $1 \%$ de fatalidade nesse raio.

Apresentadas e discutidas as consequências de todas as hipóteses levantadas, foi possível, através do programa Phast Risk ${ }^{\circledR}$ versão 6,7 desenvolvido pela DNV-Technica, obter os riscos individual e social. Para que o programa pudesse calcular esses riscos foi tomada como modelo uma sucroalcooleira do interior do estado de São Paulo. 
Tabela 3: Relação de hipóteses com suas distâncias máximas e suas respectivas condições.

\begin{tabular}{|c|c|c|c|}
\hline Hipótese & Descrição & Distância (m) & Condição \\
\hline H01 & $\begin{array}{l}\text { Grande liberação de etanol na linha de } \\
\text { 3" desde a produção até os tanques }\end{array}$ & 167,4 & $\begin{array}{c}\text { Incêndio em poça retardado ( } 1 \% \text { de } \\
\text { fatalidade) }\end{array}$ \\
\hline H02 & $\begin{array}{l}\text { Pequena liberação de etanol na linha } \\
\text { de } 3 \text { " desde a produção até os tanques }\end{array}$ & 24,9 & $\begin{array}{c}\text { Incêndio em poça retardado ( } 1 \% \text { de } \\
\text { fatalidade })\end{array}$ \\
\hline $\mathrm{H} 03$ & $\begin{array}{l}\text { Liberação de Etanol pela ruptura do } \\
\text { reservatório de } 10,000 \mathrm{~m}^{3}\end{array}$ & 169,9 & $\begin{array}{l}\text { Efeito de explosão com ignição } \\
\text { tardia ( } 1 \% \text { de fatalidade) }\end{array}$ \\
\hline H04 & $\begin{array}{l}\text { Liberação de Etanol pela ruptura do } \\
\text { reservatório de } 5.000 \mathrm{~m}^{3}\end{array}$ & 120,9 & $\begin{array}{l}\text { Efeito de explosão com ignição } \\
\text { tardia ( } 1 \% \text { de fatalidade) }\end{array}$ \\
\hline H05 & $\begin{array}{l}\text { Liberação de Etanol pela ruptura do } \\
\text { reservatório de } 3.000 \mathrm{~m}^{3}\end{array}$ & 167,4 & $\begin{array}{c}\text { Incêndio em poça retardado }(1 \% \text { de } \\
\text { fatalidade })\end{array}$ \\
\hline H06 & $\begin{array}{c}\text { Grande liberação de etanol líquido na } \\
\text { linha de } 3 \text { " desde os tanques até os } \\
\text { caminhões-tanques }\end{array}$ & 167,4 & $\begin{array}{c}\text { Incêndio em poça retardado ( } 1 \% \text { de } \\
\text { fatalidade })\end{array}$ \\
\hline H07 & $\begin{array}{c}\text { Pequena liberação de etanol líquido na } \\
\text { linha de } 3 \text { " desde os tanques até os } \\
\text { caminhões-tanques }\end{array}$ & 24,5 & $\begin{array}{c}\text { Incêndio em poça retardado }(1 \% \text { de } \\
\text { fatalidade })\end{array}$ \\
\hline H08 & $\begin{array}{l}\text { Grande liberação de etanol líquido no } \\
\text { mangote de 3" }\end{array}$ & 106,0 & $\begin{array}{c}\text { Incêndio em poça retardado ( } 1 \% \text { de } \\
\text { fatalidade) }\end{array}$ \\
\hline H09 & $\begin{array}{l}\text { Pequena liberação de etanol líquido no } \\
\text { mangote de } 3 "\end{array}$ & 19,6 & $\begin{array}{c}\text { Incêndio em poça retardado ( } 1 \% \text { de } \\
\text { fatalidade) }\end{array}$ \\
\hline H10 & $\begin{array}{l}\text { Liberação de etanol devido a ruptura } \\
\text { de caminhão-tanque (capacidade para } \\
\qquad 28 \mathrm{~m}^{3} \text { ) }\end{array}$ & 59,0 & $\begin{array}{l}\text { Incêndio em poça retardado ( } 1 \% \text { de } \\
\text { fatalidade) }\end{array}$ \\
\hline
\end{tabular}

Considerando, dessa forma, que a empresa está a uma distância de 3,5 km do centro da cidade, na qual vivem cerca de 25.000 habitantes. A Figura 2 mostra a empresa e a população considerados no programa. Dessa forma observam-se os limites da empresa e a população ao redor dela que se concentra em dois pontos A e B.

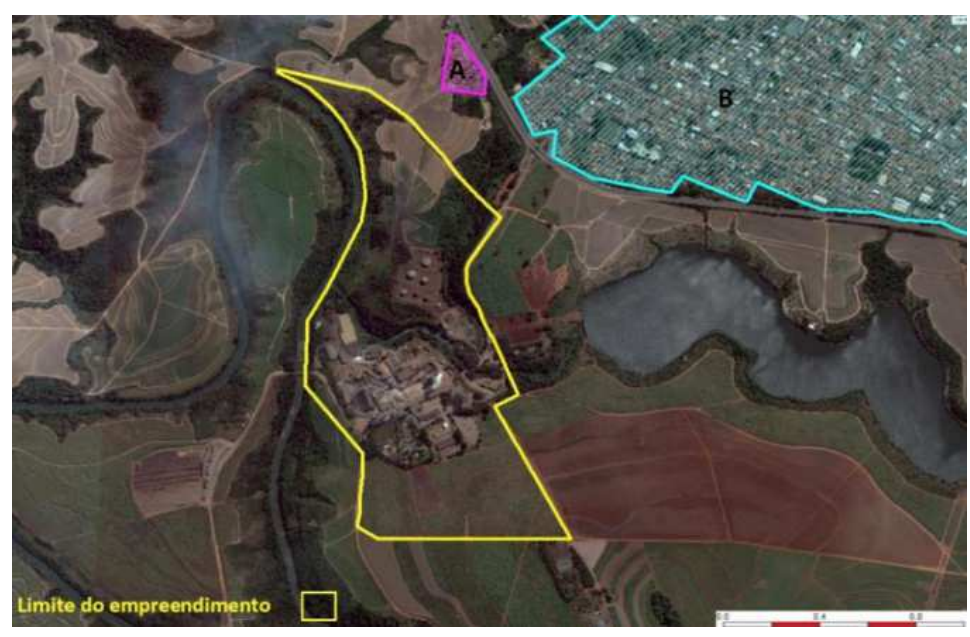

Figura 2 - Delimitação dos limites da empresa

Outro dado necessário para o cálculo do risco individual e/ou social é a frequência com que cada hipótese acontece. Para obtenção da frequência de cada hipótese, foram somadas as frequências das falhas dos componentes existentes no trecho considerado que podem gerar ou sofre uma falha e para encontrar essas taxas de falhas foi utilizado com referência o manual BEVI (2009). Na Tabela 3 são apresentadas as taxas de falha dos 
componentes considerados.

Tabela 3 - Taxa de falhas

\begin{tabular}{ccc}
\hline Componente & Tipo falha & Taxa de falha (oc/ano) \\
\hline Bomba Centrífuga & Ruptura total & $1,00 \times 10^{-05}$ \\
Bomba Centrífuga & Furo & $5,00 \times 10^{-05}$ \\
Linha com $75 \mathrm{~mm}<\mathrm{D} \leq 150 \mathrm{~mm}$ & Ruptura total & $3,00 \times 10^{-07}$ \\
Linha com $75 \mathrm{~mm}<\mathrm{D} \leq 150 \mathrm{~mm}$ & Furo & $2,00 \times 10^{-06}$ \\
Tanque atmosférico & Ruptura total & $5,00 \times 10^{-06}$ \\
Válvula de alívio & Abertura & $2,00 \times 10^{-05}$ \\
Mangote & Ruptura total & $3,50 \times 10^{-02}$ \\
Mangote & Furo & $3,50 \times 10^{-01}$ \\
Caminhão com tanque atmosférico & Ruptura total & $1,00 \times 10^{-05}$ \\
\hline
\end{tabular}

Outro fator importante a ser considerado para o cálculo das frequências de falhas dos equipamentos é o fator de utilização. Esse fator considera o quanto o equipamento é usado durante as atividades da empresa. As frequências de ocorrência das hipóteses foram determinadas pela Equação 1:

$$
\text { Fator de utilização }=\frac{n^{\mathrm{o}} \text { de operações } \times \text { tempo médio de operações }}{\text { horas do dia }}
$$

Como os dados dos pontos de liberação, com a quantidade de população ao redor e sua distância para a empresa, com as consequências simuladas e os dados de frequências de ocorrência para cada hipótese é possível através do programa quantificar-se o risco, que é apresentado na Figura 3.

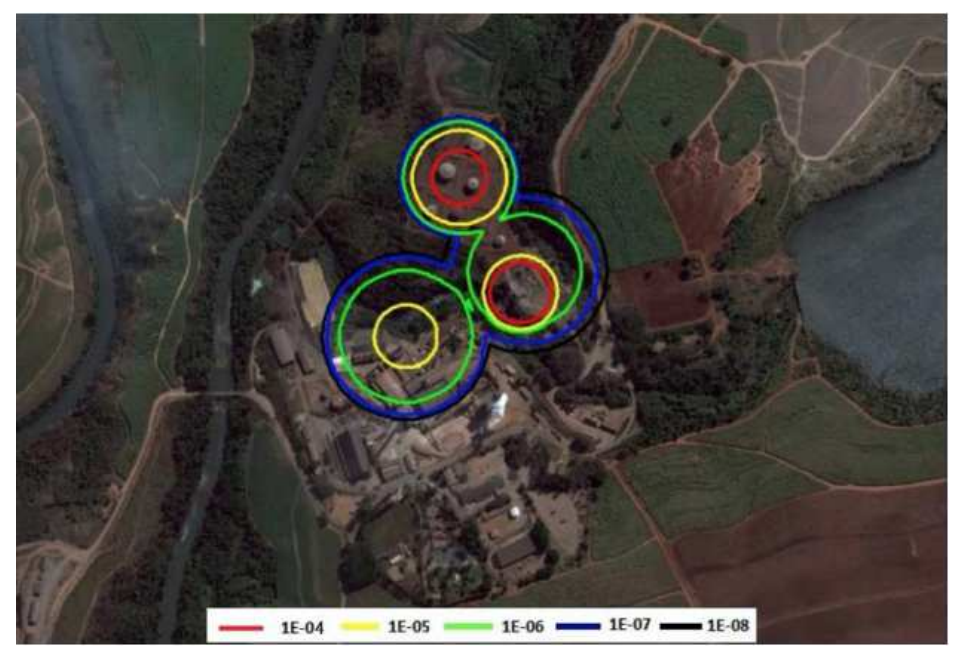

Figura 3 - Frequência de ocorrência de fatalidade

Observa-se a partir da Figura 3 os raios que representam a probabilidade de fatalidade no período de um ano, ou seja, a frequência de fatalidade que quantifica o risco. Verifica-se que nas curvas vermelhas que estão mais próximas aos pontos de liberação existe a frequência de $1 \times 10^{-04}$ da fatalidade de um indivíduo por ano. Já as curvas em preto estão mais afastadas do centro dos pontos de liberação e representam uma frequência de $1 \times 10^{-08} \mathrm{da}$ 
fatalidade de um indivíduo por ano. Também se verifica que o risco não atinge a população ao redor da empresa e então considera-se que só existe risco individual, pois risco individual é a frequência esperada que um indivíduo situado em qualquer posição (nesse caso dentro das instalações da empresa) venha a sofre uma fatalidade devido à algum acidente e não atingindo a comunidade em volta da empresa.

\section{CONCLUSÕES}

A partir do fluxograma na Figura 1, uma análise de históricos e substâncias permitiu a identificação de quais etapas são perigosas e seriam adequadas analisar mais a fundo. Dessa forma, análises qualitativas que são representadas por tabelas de APP, HAZOP e uma AEE foram levantadas com base em hipóteses resultantes no levantamento de histórico. Com isso foi realizado uma análise quantitativa usando-se o software Phast Risk ${ }^{\circledR}$ que determinou qual cenário possui a maior distância da indústria para que a área seja considerada "segura". $\mathrm{O}$ software calculou que o maior raio é de 169,9 metros para a liberação de Etanol pela ruptura do reservatório de $10.000 \mathrm{~m}^{3}$.

Tendo como base esses resultados, foi possível a realização de uma análise quantitativa para as hipóteses que envolvem o etanol. Através do software Phast Risk ${ }^{\circledR}$ e com as frequências das ocorrências das hipóteses, foi possível determinar os riscos individual e social. Os raios gerados pelo software mostram que não há risco social, há apenas individual, como pode-se ver na Figura 3. Mas como o risco individual chega a valores de $1 \times 10^{-04}$, pela classificação da CETESB, ele é considerado intolerável. Deste modo, nota-se que para esse caso adotado é necessário que haja a determinação de medidas para se diminuir o valor do risco, ou se não for possível a diminuição é precisa, hipoteticamente, que o projeto da instalação seja refeito.

\section{AGRADECIMENTOS}

Ao Centro Universitário FEI pelo suporte para o desenvolvimento do trabalho.

\section{REFERÊNCIAS}

BEVI. Reference manual bevi risk assessments. 2009. 189 p. Disponível em: http://infonorma.gencat.cat/pdf/AG_AQR_2_Bevi_V3_2_01-07-2009.pdf>. Acesso em: 14 maio 2016.

COMPANHIA ESTADUAL DE TECNOLOGIA DE SANEAMENTO AMBIENTAL CETESB. Sistema de informações sobre emergências químicas da CETESB. Disponível em: <http://sistemasinter.cetesb.sp.gov.br/emergencia/relatorio.php>. Acesso em: 15 out. 2015.

COSTA, K. Segurança de processos industriais: comissão de análise e gerenciamento de riscos. Beta $\quad$ EQ. 30 jan. 2015.2 Disponível em: http://betaeq.blogspot.com.br/2015/01/seguranca-de-processos-industriais.html>. Acesso 24 ago 2015.

LIMA, U. A.; BASSO, L. C.; AMORIN, H. V. Produção de etanol, In: LIMA, U. A.LIMA, U. A. et al. Biotecnologia Industrial: Processos fermentativos e enzimáticos. São Paulo: Edgard Blücher LTDA, 2001. v. 3, p. 1-43. 
RAUSAND, Marvin. Event Tree Analysis. Marvin Rausand and Arnljot Høyland, System Reliability Theory: Models, Statistical Methods and Applications, Second edition (Hoboken, NJ: Wiley, 2004), p. 108-117, 2005. Disponível em: <http://frigg.ivt.ntnu.no/ross/srt/slides/chapt03-eta.pdf>. Acesso em: 2 nov. 2015.

RIVM. Reference manual bevi risk assessments. Version 3.2. Bilthoven, 2009. 189p.Translation of the: Handleiding Risicoberekeningen Bevi. Versie 3.2. Disponível em:< http://infonorma.gencat.cat/pdf/AG_AQR_2_Bevi_V3_2_01-07-2009.pdf >. Acesso em: 14 maio 2016.

REVISTA BRASILEIRA DE ENGENHARIA QUÍMICA. A química da segurança. Revista Brasileira de Engenharia Química.[S.1.], v.24, n. 2, p. 7-11, 2008.

RODRIGUES, Marta Filipa Oliveira. Análise de Risco em Projectos de Construção. 2009. 54 f. Dissertação (Mestrado Integrado em Engenharia Civil) - Faculdade de Engenharia da Universidade do Porto, Portugal.

SOUZA, C. R. C. Análise e gerenciamento de riscos de processos industriais. Niterói, RJ. Universidade Federal Fluminense. [200-]. Disponível em: $<$ http://www.areaseg.com/bib/12\%20\%20Arquivos\%20Diversos/Apostila_de_Gerencia mento_de_Riscos.pdf $>$. Acesso em 27 ago. 2015.

SOUZA, E. A. O treinamento industrial e a gerência de riscos: uma proposta de instrução programada. 1995. 126p. Dissertação (Mestrado em Engenharia de Produção). Escola de Engenharia de Produção e Sistemas, Universidade Federal de Santa Catarina, Florianópolis.

THE INTERNATIONAL BANK FOR RECONSTRUCTION AND DEVELOPMENT -THE WORLD BANK. Técnicas para avaliação de perigos industriais: um manual. 2. ed. Washington, D.C.: Technica LTD, 1990. Disponível em: <http://wwwwds.worldbank.org/servlet/WDSContentServer/WDSP/IB/2004/01/27/000 178830_98101904165042/Rendered/PDF/multi0page.pdf>. Acesso em 23 mar. 2016. 


\title{
ANALYSIS OF RISKS AND CONSEQUENCES IN A SUGAR-ALCOHOL PLANT
}

\author{
M.P.A. MARIN ${ }^{1, *}$, G.M. DE MELO ${ }^{1}$, G.B.H. DE PAULO ${ }^{1}$, L.F.M. LABATE ${ }^{1}$, \\ T.B. MIRANDA ${ }^{1}$, V. SCHINCARIOL ${ }^{1}$ \\ ${ }^{1}$ FEI University, Department of Chemical Engineering \\ *E-mail: marimari@fei.edu.br
}

\begin{abstract}
The existing risks of the sugar-alcohol industry were analyzed by dividing the process into blocks and identifying the main risks, as well as the removal of dangerous substances from the process, mainly anhydrous alcohol, hydrated alcohol and sugar, due to the large volume in the process and its physical-Chemical properties. It was verified that $90 \%$ of the accidents involving ethanol occur in road transport and $2 \%$ in industry and storage. Risk analysis was carried out in product storage and transportation. PHAs, ETA and Hazops were applied as qualitative methods of analysis. The Phast ${ }^{\circledR}$ software showed that the largest range was 169.9 meters in the release of Ethanol by rupturing the $10,000 \mathrm{~m}^{3}$ reservoir. The social and individual risks were also obtained. The results indicate that fatalities will occur only within the industry, just indicating individual risk. This risk reaches a maximum of 1E-04, considered intolerable by CETESB.
\end{abstract}

KEYWORDS: Risk analysis; Hazards identification; Hazop; PHA; ETA 